Revista de Estudios Histórico-Jurídicos

[Sección derecho romano]

XLII (Valparaíso, Chile, 2020)

[pp. 97-143]

\title{
El Digesto y su Definición DE LiberTad
}

[The Digest and its Definition of Freedrom]

\author{
Carlos Amunátegui Perelló* \\ Pontificia Universidad Católica de Chile
}

\begin{abstract}
RESUMEN
La legislación justinianea contiene una singular definición de libertad, la facultad de hacer lo que se desee, salvo que la fuerza o el Derecho lo impidan (D. 1, 4, 5 pr. y IJ. 1, 3, 1). Dicha categoría desciende directamente de la filosofía estoica y, aunque la academia ha tendido a pasarla por alto, creemos contiene importantes elementos provenientes de la filosofía política tardorepublicana relativos a la limitación del poder y el rol del Derecho frente a ella.
\end{abstract}

\section{Palabras clave}

Libertad - esclavitud - Digesto - Florentino - derecho - hechos.
ABSTRACT

Justinan's legislation holds a singular definition of liberty, the faculty to do as one wishes, except when force or law forbids it (D. 1, 4, 5 pr. and IJ. 1, 3, 1). Such concept comes directly from stoic school of thought and, although academy has usually passed swiftly over it, we believe it contains some important elements which come from late Republican political philosophy about limitation of power and the role of law.

$$
\begin{aligned}
& \text { KEY WORDS } \\
& \text { Liberty - freedom - slavery - Digest } \\
& \text { - right - facts }
\end{aligned}
$$

RECiBiDO el 16 de abril de 2020 y ACEPTADO el 9 de junio de 2020

\section{INTRODUCCIÓN}

La libertad es uno de los conceptos fundamentales del derecho moderno. Se encuentra presente en todas las constituciones modernas, jugando diversos roles en el sistema positivo, y usualmente se la incluye entre los derechos fundamentales, siguiendo la tradición liberal del siglo XVIII.

En las famosas declaraciones de derechos del siglo XVIII, la libertad se fundamentaba en el derecho natural, una idea que viene directamente del pasado clásico, repetida muchas veces en el Digesto de Jutiniano y probablemente elaborada durante la República tardía y el Alto Imperio. Así, por ejemplo, en el artículo $4^{\circ}$

* Profesor de Derecho Romano, Pontificia Universidad Católica de Chile. Este artículo es parte del proyecto Fondecyt 1180022. Dirección postal: Piso 4º, Facultad de Derecho, Pontificia Universidad Católica de Chile, Alameda 340, Santiago. Correo electrónico: camunate@uc.cl. 
de la Declaración Universal de Derechos del Hombre y del Ciudadano de 1789 se lee: "La liberté consiste à pouvoir faire tout ce qui ne nuit pas à autrui : ainsi, l'exercice des droits naturels de chaque homme n'a de bornes que celles qui assurent aux autres Membres de la Société la jouissance de ces mêmes droits. Ces bornes ne peuvent être déterminées que par la Loi".

El artículo, como veremos, se inspira directamente en la definición de Florentino de libertad, contenida en D. 1, 5, 4, haciendo expresa referencia a la doctrina de los derechos naturales.

No obstante, el concepto de libertad es tratado de manera desigual en las fuentes romanas. Hay poca información acerca del rol que jugaba en el Derecho público. Aunque algunos académicos han identificado el concepto de libertad como una suerte de suma de derechos ${ }^{1}$, compuesta de diversos poderes que tenía un ciudadano, la mayor parte del material con que contamos no menciona tal concepto. Ciertamente, la ciudadanía comprendía diferentes capas de protección en contra del actuar arbitrario del poder ${ }^{2}$, y la facultad de participar en los asuntos políticos de la República ${ }^{3}$, pero la libertas no era lo mismo que la ciudadanía. La noción de libertad parece estar implicada en la de ciudadanía, como la libertad común de que los miembros de la ciudad gozan. Esta noción de libertas parece jugar un rol importante en la arena política, regulando la relación entre el ciudadano y el gobierno ${ }^{4}$, especialmente en la República tardía, aunque tiende a desaparecer en el Principado 5 . Resulta interesante notar que el concepto jurídico de libertad tiende a no enfatizar esta noción "pública" de libertad, sino que parece enfocarse en una idea relacionada, aunque algo distinta, libertas como no servidumbre ${ }^{6}$, un

${ }^{1}$ Volterra, Edoardo, Manomissione e cittadinanza, en: Talamanca, Mario (ed.), Scritti Giuridici (Napoli, 1991), II, p. 410. En igual sentido WirszuBsKI, Charles, 'Libertas' as a Political Idea During the late Republic and Early Empire (Cambridge, 1950), pp. 7-30 y Franciosi, Gennaro, Rezension a Wirszubski, en SDHI., 20 (1953), pp. 330-333.

${ }^{2}$ Como son la provocatio y el auxilium de los tribunos.

${ }^{3}$ Brunt, Peter A., The Fall of the Roman Republic and Related Essays (Oxford, 1988), p. 297. Acerca del problema, véase también Manfredini, Arrigo Diego, Le pilleus libertatis (C. 7 , 2, 10 - C. 7, 6, 1, 5), en RIDA., 57 (2010), pp. 247-263; Bauman, Richard A., Human Rights in Ancient Rome (London-New York, 2000), p. 101; ArenA, Valentina, Libertas and the Practice of Politics in the Late Roman Republic (Cambridge, 2012), p. 54.

${ }^{4}$ Por ejemplo, Arena abre su libro con una definición funcional del concepto: "I am exclusively concerned with the notion of political liberty, understood as the relation between the liberty of the citizen and the power of the commonwealth, and its conflicting applications in the political debates that took place in the last period of the Republic [...]" ARENA, Valentina, cit. (n. 2), p. 1.

${ }^{5}$ Diversos aspectos de la libertad fueron eliminados durante el Principado. Así, por ejemplo, "freedom of speech, libertas, especially among the elite, to all intents and purposes disappeared: freedom as the old Republic knew it was swept away and Rome's Senate, which for centuries had shaped policy and presided over the growth of Mediterranean empire, was reduced to a state of servitude -as even emperors complained (Ann.3,65)" BRADlEY, Keith, Freedom and Slavery, en BARCHIESI, Alessandro y SCHEIDEL, Walter (eds.) The Oxford Handbook of Roman Studies (Oxford, 2010), p. 624. En palabras de Kunkel: "im Munde der Kaiser bedeutet libertas überhaut nicht mehr die echte republikanische Freiheit, sondern ungefärh das gleiche wie securitas". KUNKEL, Wolfgang, Bericht über neuere Arbeiten zur römischen Verfassungsgeschichte. III, en ZRG RA., 73/1 (1956), p. 323.

${ }^{6}$ El lugar más famoso, aunque no exclusivo: Gai. 1, 9; D. 1, 1, 4; D. 1, 5, 3-5; D. 12, 6, $64 \mathrm{y}$, por último, IJ. 1, 3, 1. 
estatuto de no dominación en el sentido que el individuo no es un esclavo ${ }^{7}$. Esto apunta a un concepto negativo de libertad, el que tiene un significado jurídico preciso y que parece primario en la construcción de las ideas romanas acerca de la libertas $^{8}$, incluso en su forma más compleja y pública. Exploraremos la noción de libertad en el Derecho romano en las siguientes secciones de este texto a través del análisis de una inquietante definición contenida en el Digesto, donde se define la libertad como el poder de hacer lo que a uno le plazca.

\section{LA ESCLAVITUD Y LA LIBERTAD POLÍTICA}

Durante el siglo III a.C., Roma concluyó su expansión en la península itálica y comenzó su lucha por la dominación de la cuenca mediterránea. Su recién descubierta predominancia llegó de la mano de importantes victorias militares que permitieron la conversión de ejércitos derrotados en una provisión fresca de mano de obra. No podemos obtener una cifra precisa acerca del número de esclavos en Roma en ninguna época, aunque algunas estimaciones han sido realizadas 9 que tienden a elevar la cifra para la península itálica a entre un veinte y un treinta porciento de la población. Apiano describe la situación en los siguientes términos ${ }^{10}$ : "Por estas razones, los ricos se hicieron máximamente opulentos y los esclavos abundaban en el campo, mientras el hambre y la escasa población afligían al pueblo italiano, diezmado por la pobreza, los impuestos y el servicio militar".

La población podía ser dividida de una manera simple entre libres y esclavos, puesto que esta era la diferencia capital entre la fuerza de trabajo sujeta y el resto de la población, que se beneficiaba de la posición imperial que Roma había adquirido. Los libres contaban con una esfera de sacro-santidad, asociada con los derechos políticos que la plebe había obtenido en las luchas de la época precedente. El auxilium de los tribunos, la provocatio ad populum y la posibilidad de participar en el gobierno de la ciudad a través del voto se hicieron los atributos esenciales que configuraban el estatuto del ciudadano libre que, por consiguiente, vivía en una república igualmente libre ${ }^{11}$. La libertas se convirtió en un concepto político y su desarrollo ha sido objeto de un profundo desarrollo en las investigaciones

${ }^{7}$ Galinsky, Karl, Vergil's Uses of "Libertas": Texts and Contexts, en Vergilius, 52 (2006), p. 6; Arena, Valentina, cit. (n. 2), p. 14; Strunk, Thomas E., History after Liberty. Tacitus on Tyrants, Sycophants and Republicans (Michigan, 2017), p. 23.

${ }^{8}$ Levy, Ernst, Libertas und Civitas, en ZRG RA., 78 (1961), p. 142; VON LÜBTow, Ulrich, Blüte und Verfall der römischen Freiheit (Berlin, 1953), p. 20.

${ }^{9}$ El más importante: SCHEIDEL, Walter, Human Mobility in Roman Italy, II: The Slave Population, en The Journal of Roman Studies, 95 (2005), pp. 64-79. No obstante, la cantidad de personas libres en Italia parece perturbadoramente alta. Tres cuartos de todas las lápidas mencionan el estatuto de liberto de la persona inhumada. Véase: MouriTsEn, Henrik, Freedmen and Decurions: Epitaphs and Social History in Imperial Italy, en The Journal of Roman Studies, 95 (2005), pp. 38-63.

10App. BC 1, 1, 7, 28.

${ }^{11}$ Strunk, Thomas E., cit. (n. 7), p. 23; Bleicken, Jochen, Der Begriff der Freiheit in der letzten Phase der Römischen Republik, en Historische Zeitschrift, 195 (1962), pp. 1-20; KunKEL, Wolfgang, cit. (n. 5), pp. 302-352. 
de la academia reciente. No obstante, el concepto de libertas permaneció inexorablemente ligado al de esclavitud. En el Digesto de Justiniano encontramos una interesante definición de libertas": "Florentino, libro noveno de las instituciones. La libertad es la facultad natural de hacer lo que se desee, salvo si está prohibido por la violencia o el Derecho". Esta definición, repetida textualmente en las Instituciones de Justiniano ${ }^{13}$, no ha merecido la atención de los estudios recientes, siendo incluso descartada como una "una banale riminiscenza di dottrine stoiche" 14 . No obstante, pensamos que contiene algunos elementos interesantes que debiesen ser tomados en cuenta al escribir tanto sobre la libertad política como sobre su sentido jurídico.

La definición está obviamente tomada de la tradición filosófica estoica. De hecho, Cicerón da cuenta de ideas similares en su paraxoda stoicorum ${ }^{15}$ : “¿Qué es la libertad? El poder de hacer lo que se desee”. El autor desarrolla el concepto señalando que el hombre sabio es quien ejerce la auténtica libertad, puesto que actúa sin estar constreñido por la fuerza o el derecho, sino por su propio albedrío ${ }^{16}$. No es de extrañar que el concepto jurídico de libertas se desarrolle a partir de teorías estoicas, puesto que la discusión respecto a la esclavitud en el derecho romano estuvo profundamente influenciada por esta escuela ${ }^{17}$. Tenemos muchos ejemplos en la materia, incluyendo entre los ejemplos las famosas críticas a la esclavitud que pueden ser encontradas en el Digesto ${ }^{18}$.

Respecto a Florentino, tenemos muy poca información disponible. Parece haber vivido en la primera mitad del siglo III, toda vez que él copia un texto del jurista Paulo $^{19}$. Como es esperable de un hombre culto de su época, conocía el

${ }^{12}$ D. 1, 5, 4 "Florentinus libro nono institutionum. pr. Libertas est naturalis facultas eius quod cuique facere libet, nisi si quid vi aut iure prohibetur".

${ }^{13}$ IJ. 1, 3, 1.

${ }^{14}$ BetTI, Emilio, Istituzioni di diritto romano (Padova, 1943), p. 41.

${ }^{15}$ Cic. Parad. 34 "Quid est enim libertas? Potestas vivendi, ut velis".

${ }^{16}$ Cic. Parad. 34 "Soli igitur hoc contingit sapienti, ut nihil faciat invitus, nihil dolens, nibil coactus".

${ }^{17}$ Mitsis, Philip, The Stoic Origin of Natural Rights, en Ierodiakonou, K. (ed.) Topics in Stoic Philosophy (Oxford, 1999), pp. 153-177; MAYER-Maly, Theo, Das “ius Gentium” bei den späteren Klassikern, en IURA, 34 (1983), pp. 91-102; LEVY, Ernst, Natural Law in Roman Thought, en SDHI, 15 (1949), pp. 3-19; KUEBLER, Bernhard, Griechische Einflüsse auf die Entwicklung der römischen Rechtswlssenschaft gegen ende der republicanischen Zeit, en Atti del congreso internazionale di diritto romano. Bologna e Roma XVII-XXVII aprile XCMXXXIII (Pavia, 1934), I, pp. 79-98; GILTAI, Jacob y TuORI, Kaius, Human Rights in Antiquity? Revisiting Anachronism and Roman Law, en SlotTe, P. (ed.) Revisiting the Origins of Human Rights (Cambridge, 2015), pp. 39-63; Honoré, Tony, Ulpian Pioneer of Human Rights (Oxford, 2002), p. 80; GaUdemeT, Jean, Des "droits de l'homme" ont-ils été reconnus dans l'empire romain?, en Labeo, 33 (1987), pp. 7-23; Él mismo, Des “droits de l'homme" dans l'antiquité?, en FeEnstra, R. et al. (eds.) Collatio iuris romani Études dédiées à Hans Ankum (Amsterdam, 1995), pp. 105-115; Honoré, Tony, Ulpian, Natural Law and Stoic Influence, en Tijdschrift voor Rechtsgeschiedenis, 78 (2010), pp. 199-208; Brouwer, René, Ulpian's Appeal to Nature: Roman Law as Universal Law, en Tijdschrift voor Rechtsgeschiedenis, 60 (2015), pp. 60-76; CASTELlo, Carlo, Humanitas e favor libertatis nel I secolo, en Sodalitas. Scritti in onore di Antonio Guarino (Napoli, 1984), V, pp. 2175-2190.

${ }^{18}$ D. 1, 1, 4; D. 1, 5, 4; D. 40, 11, 2; D. 50, 17, 32.

${ }^{19} \mathrm{El}$ texto es D. 38, 2, 28. Vid. Lenel, Otto, Palingenesia iuris civilis (Leipzig, 1939), I, pp. 171-172; Honoré, Tony, cit. (n. 17), p. 88. 
griego y citó textos en dicha lengua ocasionalmente ${ }^{20}$. Sabemos que escribió sus propias Institutiones en doce libros, de las que 41 fragmentos son citados en el Digesto, pero contamos con poca más información respecto a él.

$\mathrm{Al}$ analizar la definición, encontramos algunos elementos extraños, como el hecho que la libertad sea concebida como una facultad, un poder. En el pensamiento jurídico romano no existía una teoría de los derechos subjetivos ${ }^{21}$, por lo que al designar la libertas como una facultas, Florentino parece estarse alejando del derecho y entrando en un terreno diferente de las relaciones sociales. No obstante, esta no es la única rareza que contiene la definición, toda vez que los límites que impone al concepto de libertad son aún más extraños. La libertad, de acuerdo a la definición, tendría dos fronteras, el derecho (iure) y la violencia (vi). El primer límite, el derecho, tiene una larga tradición académica a sus espaldas. Cicerón afirma que libertas in legibus consistit ${ }^{22}$, en el sentido que la libertad provendría del derecho ${ }^{23}$. La libertad sería el poder de actuar dentro del marco dado el derecho, la cual, en sí misma, no parece una aserción problemática. Más interesante es el segundo límite que Florentino impone a la libertas: la violencia. La violencia está fuera del marco jurídico, y confrontándola al derecho, parece que Florentino se refiere al uso ilegítimo de la fuerza. Esto es algo que ya aparece implícito en Cicerón en su Paradoxa stoicorum, cuando define el acto libre como aquél opuesto al forzado (nihil coactus). La idea es coherente, puesto que un hombre legítimamente condenado por el juez está tan privado de su libertad como aquel que se encuentra secuestrado. Sea como fuere, la consideración de que la fuerza ilegítima es contraria a la libertad parece interesante, toda vez que implica que la libertad no es un concepto jurídico, sino uno meta-jurídico. Los derechos no pueden ser afectados mediante la violencia. Todo lo contrario, si alguno es privado por la fuerza de algo que le pertenece de acuerdo al derecho, un conjunto de mecanismos jurídicos se activan a fin de prevenir la situación y restituir el imperio del derecho. El ladrón no priva a su víctima de su propiedad, sino sólo de su posesión, y a fin de recuperar tal posesión la víctima es provista de actiones in rem (reivindicatio) e in personam (actio furti). La propiedad es un derecho definido por el ordenamiento jurídico y, por tanto, no puede ser afectado por los hechos. Los aspectos fácticos del control de una cosa están comprendidos en la posesión, que no es un derecho y tiene una consistencia fáctica. Curiosamente, la libertad puede ser afectada tanto por el derecho como por los hechos. Esto trae a colación la famosa falacia naturalista de Hume ${ }^{24}$, que señala que de un hecho ("an is"), no puede deducirse una consecuencia normativa ("an ought"). Si la libertad resulta

\footnotetext{
${ }^{20}$ Véase, por ejemplo, D. 11, 7, 4.

${ }^{21}$ VILLeY, Michel, Le droit et les droits de l'homme (Paris, 1983), p. 63. Más concluyente y profundo, Guzmán Brito, Alejandro, Para la historia del derecho subjetivo, en Revista Chilena de Derecho, 2 (1975) pp. 55-68 y ÉL MISMO, Historia de la denominación del derecho-facultad como subjetivo, en REHJ., 25 (2003) pp. 407-443.

${ }^{22}$ Cic. De. L. Agr. 2.202. Acerca del problema, véase Bleicken, Jochen, cit. (n. 11), p. 1.

${ }^{23}$ Cic. De. Leg. 3.39 "Quam ob rem lege nostra libertatis species datur, auctoritas bonorum retinetur, contentionis causa tollitur".

${ }^{24}$ El término falacia naturalista viene de George Edward Moore, vid. Moore, George E., Principia Ethica (Cambridge, 1953), p. 10.
} 
afectada por los hechos, como la violencia, por tanto, no puede ser un derecho, sino algo completamente diverso.

Aunque los romanos no desarrollaron la falacia naturalista y su estricta separación entre derechos y hechos, la idea que los hechos no pueden afectar derechos está presente en su razonamiento jurídico ${ }^{25}$. Paulo señala explícitamente que los hechos no pueden afectar derechos (res facti infirmari iure civil non potest) ${ }^{26}$, implicando que las instituciones jurídicas se encuentran separadas de los fenómenos fácticos. Este razonamiento está también presente en Celso (facti, non iuris est quaestio, en D. 24, 1, 47) ${ }^{27}$ y en Juliano (facti magis quam iuris quaestio est, en D. 39, 5, 2, 7). Es más, razonamientos similares pueden ser encontrados en Marciano (D. 29, 7, 7, 1 y D. 22, 1, 32 pr, citando una constitución imperial de Antonino Pío), Papiniano (D. 41, 5, 12, 12) y Ulpiano (D. $1,5,16)^{28}$, por lo que podemos decir que la separación entre derechos y hechos estaba presente en los razonamientos jurídicos romanos. La definición de libertad de Florentino es incluso más extraña dado el contexto en que se entrega. Se la menciona a propósito del status libertatis, que es una institución complemente jurídica. De hecho, el secuestro no cambia el status libertatis de la víctima. La víctima retiene su estado de libertad, aunque no pueda ejercer tal libertad. A fin de defenderla, se la dota con la vindicatio in libertatem, para establecer su estado de libertad y recobrar el ejercicio de la misma. Esta es una situación común en Roma, omnipresente en las tramas de las comedias ${ }^{29}$ y en su pasado mitológico, como en el caso de Virginia.

\section{LIBERTAD PÚBLICA}

En cierto sentido, la definición dada por Florentino excede la materia relativa al status libertatis y parece tener un ámbito más amplio y no bien conexo con la esclavitud. Esto puede deberse a algún proceso de resumen ocurrido durante la compilación, o tal vez a la omisión de la explicación de la conexión entre la definición de libertad, contenida en D. 1, 5, 4 pr. y la definición de esclavitud de D. 1, 5, 4, 1, no podemos saberlo. ¿Significa esto que la definición es irrelevante para cualquier fin jurídico? Por supuesto que no. Existen otras materias donde la libertad es importante y la definición de Florentino parece iluminadora. Por ejemplo, en lo relativo al vicio de fuerza (metus), esto es la violencia ejercida a fin

${ }^{25}$ Mayer-Maly, Theo, Juristische Reflexionen über ius I, en ZRG RA., 117 (2000), p. 15.

${ }^{26}$ D. 41, 2, 1, 4 [Paulus libro 54 ad edictum] "Si vir uxori cedat possessione donationis causa, plerique putant possidere eam, quoniam res facti infirmari iure civili non potest: et quid attinet dicere non possidere mulierem, cum maritus, ubi noluit possidere, protinus amiserit possessionem?".

${ }^{27}$ De hecho, Celso desecha el problema de las impensae cuando éste es claramente fáctico y no jurídico: facti, non iuris est quaestio en D. 24, 1, 47.

${ }^{28}$ Para el análisis véase MAYER-Maly, Theo, cit. (n. 25), pp. 1-29.

${ }^{29}$ En efecto, uno de los elementos más característicos de la comedia plautina es la presencia de personajes femeninos que se presentan como esclavos, pero que en su infancia han sido raptados, por lo que son técnicamente libres. En la conclusión se descubre su origen y se vuelve reestablece su liberatd, lo que sirve para que el protagonista pueda contraer matrimonio con ellas. Un ejemplo típico lo constituye Anterástile, en Poenulus. Ella es una muchacha esclava respecto de quien se descubre que en verdad es libre e hija de Hanón, un cartaginés que llega a la ciudad. 
de obtener el asentimiento de una persona en un acto. La in integrum restitutio provista por el pretor en caso de metus busca proteger la libertad de la persona cuyo asentimiento ha sido constreñido por la fuerza. Este razonamiento es exactamente aquél entregado por Paulo respecto a la actio hereditatem ${ }^{30}$. Ulpiano, citando a Pomponio respecto al interdicto unde vi, declara que el objetivo de la institución es liberar a la víctima de la violencia, sea que venga de los enemigos, de los ladrones o incluso de un populum ${ }^{31}$ (si quo magis te de vi hostium vel latronum vel populi tuerer vel liberarem). Resulta interesante que actos privados de violencia sean puestos en conjunto con actos públicos, y que el interdicto otorgue protección respecto a ambos.

Esto parece algo extraño, toda vez que relaciona la noción de libertad con un nivel más alto de pensamiento y no meramente con el status libertatis, sino con una concepción general de libertad que envuelve tanto actos públicos como privados. En este sentido, Marciano señala explícitamente que la libertad no es un asunto privado, sino público ${ }^{32}$ (libertatem non privata, sed publica res est), lo que nos lleva directo a las discusiones relativas a la libertad pública en la República tardía.

La libertad de la República puede estar constreñida por la violencia, como en el caso en que un grupo revolucionario tome el control del aparato público. Esto parece estar implicado en la declaración inicial de Augusto en sus Res Gestae ${ }^{33}$ : "Vindiqué la Republica a la libertad, la que estaba oprimida por la dominación de las facciones". Pero, nuevamente, la libertad tiene dos límites, vi y el ius, lo que lleva a preguntarse, si la libertad tiene límites fácticos, ¿tendrá también límites jurídicos? ¿Pueden haber límites a los actos públicos? ¿Existe algún tipo de límite constitucional al poder de la civitas? Recientemente, Strauman ${ }^{34}$ ha escrito extensamente acerca del constitucionalismo en la República tardía y ha concluido que se desarrolló un esfuerzo de diversos intelectuales, Cicerón entre ellos, para construir una suerte de frontera a la acción de los comicios, limitando el poder del populus para actuar en contra de la libertad de la República, especialmente en la concesión de poderes extraordinarios a individuos, en último término fundamen-

${ }^{30}$ D. 4, 2, 21, 5 [Paulus libro 11 ad edictum] "Si metu coactus adii hereditatem, puto me heredem effici, quia quamvis si liberum esset noluissem, tamen coactus volui: sed per praetorem restituendus sum, ut abstinendi mihi potestas tribuatur".

${ }^{31}$ D. 4, 2, 9, 1 [Ulpianus libro 11 ad edictum] "Animadvertendum autem, quod praetor hoc edicto generaliter et in rem loquitur nec adicit a quo gestum: et ideo sive singularis sit persona, quae metum intulit, vel populus vel curia vel collegium vel corpus, huic edicto locus erit. Sed licet vim factam a quocumque praetor complectatur, eleganter tamen Pomponius ait, si quo magis te de vi hostium vel latronum vel populi tuerer vel liberarem, aliquid a te accepero vel te obligavero, non debere me hoc edicto teneri, nisi ipse hanc tibi vim summisi: ceterum si alienus sum a vi, teneri me non debere, ego enim operae potius meae mercedem accepisse videor".

${ }^{32}$ D. 40, 5, 53 pr. "Marcianus libro quatro regularum. Si quis rogatus ancillam manumittere moram fecerit, si interea enixa fuerit, constitutum est huiusmodi partum liberum nasci et quidem ingenuum. Sed sunt constitutiones, quibus cavetur statim ex quo libertas deberi coeperit ingenuum nasci: et hoc magis est sine dubio sequendum, quatenus libertas non privata, sed publica res est, ut ultro is qui eam debet offerre debeat".

${ }^{33}$ Aug. Res Gestae 1 "rem publicam a dominatione factionis oppressam in libertatem vindicavi”.

${ }^{34}$ Straumann, Benjamin, Crisis and Constitutionalism. Roman Political Thought from the Fall of the Republic to the Age of Revolution (Oxford, 2016), pp. 23-147. 
tando estos límites en el derecho natural. Dada la naturaleza poco representativa de las asambleas romanas y la facilidad con que las leges se aprobaban en el sistema republicano ${ }^{35}$, la construcción de límites jurídicos a los poderes aparentemente ilimitados de los comitia parece coherente. En cualquier caso, respecto a cualquier teorización relativa a la libertad pública que haya sido desarrollada en la República tardía, sólo tenemos fugaces reflejos en las fuentes. La definición de Florentino puede, en efecto, ser otro reflejo, aunque esté extrañamente incluida en el acápite relativo al status libertatis, una materia para la cual, tal vez, no haya sido inicialmente diseñada.

\section{BiBLIOGRAFÍA}

Arena, Valentina, Libertas and the Practice of Politics in the Late Roman Republic (Cambridge, 2012).

Bauman, Richard A., Human Rights in Ancient Rome (London-New York, 2000).

BETTI, Emilio, Istituzioni di diritto romano (Padova, 1943).

BLEICKEN, Jochen, Der Begriff der Freiheit in der letzten Phase der Römischen Republik, en Historische Zeitschrift, 195 (1962), pp. 1-20.

Bradley, Keith, Freedom and Slavery, en BARChiesi, Alessandro y Scheidel, Walter (eds.) The Oxford Handbook of Roman Studies (Oxford, 2010), pp. 624-636.

Brouwer, René, Ulpian's Appeal to Nature: Roman Law as Universal Law, en Tijdschrift voor Rechtsgeschiedenis, 60 (2015), pp. 60-76.

Brunt, Peter A., The Fall of the Roman Republic and Related Essays (Oxford, 1988).

CASTEllo, Carlo, Humanitas e favor libertatis nel I secolo, en Sodalitas. Scritti in onore di Antonio Guarino (Napoli, 1984), V, pp. 2175-2190.

Franciosi, Gennaro, Rezension a WirszUbSKI, en SDHI., 20 (1953), pp. 330-333.

Galinsky, Karl, Vergil's Uses of "Libertas": Texts and Contexts, en Vergilius, 52 (2006), pp. 3-19.

GaUdemet, Jean, Des "droits de l'homme" ont-ils été reconnus dans l'empire romain?, en Labeo, 33 (1987), pp. 7-23.

—Des "droits de l'homme" dans l'antiquité?, en FeEnstra, R. et al. (eds.) Collatio iuris romani Études dédiées à Hans Ankum (Amsterdam, 1995), pp. 105-115.

Giltai, Jacob y TuORI, Kaius, Human Rights in Antiquity? Revisiting Anachronism and Roman Law, en SlOtTe, P., (ed.) Revisiting the Origins of Human Rights (Cambridge, 2015), pp. 39-63.

Honoré, Tony, Ulpian Pioneer of Human Rights (Oxford, 2002).

-Ulpian, Natural Law and Stoic Influence, en Tijdschrift voor Rechtsgeschiedenis, 78 (2010), pp. 199-208.

KUEBLER, Bernhard, Griechische Einflïsse auf die Entwicklung der römischen Rechtswlssenschaft gegen ende der republicanischen Zeit, en Atti del congreso internazionale di diritto romano. Bologna e Roma XVII-XXVII aprile XCMXXXIII (Pavia, 1934), I, pp.79-98.

KUNKEL, Wolfgang, Bericht über neuere Arbeiten zur römischen Verfassungsgeschichte. III, en $Z R G R A ., 73 / 1$ (1956), pp. 302-352.

Lenel, Otto, Palingenesia iuris civilis (Leipzig, 1939), I.

${ }^{35}$ Mouritsen, Henrik, Politics in the Roman Republic (Cambridge, 2017), p. 57. 
Levy, Ernst, Natural Law in Roman Thought, en SDHI, 15 (1949), pp. 3-19.

—Libertas und Civitas, en ZRG RA., 78 (1961), pp. 142-172.

Manfredini, Arrigo Diego, Le pilleus libertatis (C. 7, 2, 10 - C. 7, 6, 1, 5), en RIDA. 57 (2010), pp. 247-263.

Mayer-Maly, Theo, Das "ius Gentium" bei den späteren Klassikern, en IURA. 34 (1983), pp. 91-102.

-Juristische Reflexionen über ius I, en ZRG RA., 117 (2000), pp. 1-29.

Mitsis, Philip, The Stoic Origin of Natural Rights, en IerodiaKonou, K. (ed.) Topics in Stoic Philosophy (Oxford, 1999), pp. 153-177.

Moore, George E., Principia Ethica (Cambridge, 1953).

Mouritsen, Henrik, Freedmen and Decurions: Epitaphs and Social History in Imperial Italy, en The Journal of Roman Studies, 95 (2005), pp. 38-63.

-Politics in the Roman Republic (Cambridge, 2017).

SCHeIDel, Walter, Human Mobility in Roman Italy, II: The Slave Population, en The Journal of Roman Studies, 95 (2005), pp. 64-79.

Straumann, Benjamin, Crisis and Constitutionalism. Roman Political Thought from the Fall of the Republic to the Age of Revolution (Oxford, 2016).

STRUnK, Thomas E., History after Liberty. Tacitus on Tyrants, Sycophants and Republicans (Michigan, 2017).

Volterra, Edoardo, Manomissione e cittadinanza, en TAlamanca, Mario (ed.), Scritti Giuridici (Napoli, 1991), II.

VON LÜвTOW, Ulrich, Blüte und Verfall der römischen Freiheit (Berlin, 1953).

VILLEY, Michel, Le droit et les droits de l'homme (Paris, 1983).

Wirszubski, Charles, Libertas as a Political Idea During the late Republic and Early Empire (Cambridge, 1950). 